\title{
Assessment of the Performance of Frequency Domain Models Based on Different Reference Points for Linearization
}

\author{
E. Tavukcu, S. Müller, J. Meyer \\ Technische Universitaet Dresden \\ Institute of Electrical Power Systems and High Voltage Engineering \\ 01062 Dresden (Germany) \\ Phone number: 0049351463 43201, e-mail: ebru.tavukcu@tu-dresden.de, sascha.mueller@tu-dresden.de
}

\begin{abstract}
Power electronic-based loads are increasingly used in modern distribution systems. Such appliances inject harmonic currents into the power system and thus, they can have serious impacts on the power quality of the system. Therefore, it is necessary to find suitable frequency domain models of these loads to predict their contribution to the harmonic voltage levels. So far, various modeling approaches have been proposed in the relevant literature. Usually, they are parameterized based on laboratory measurements. In this paper, however, a circuit-based time domain model of a Compact Fluorescent Lamp (CFL) is implemented in MATLAB $® / S i m u l i n k ®$ and used for the parameter identification of three different frequency domain models. These models usually rely on a linearization of their emission characteristic and therefore, it is required to choose an appropriate reference voltage. As the supply voltage in real distribution systems is already distorted, the commonly made assumption of a sinusoidal reference voltage may increase the inaccuracy of models for strongly non-linear devices. Therefore, different reference points for the model parameterization are specified and their impact on the model performance under typical voltage distortions is studied.
\end{abstract}

\section{Key words}

Harmonic modeling, nonlinear load, frequency-domain analysis, harmonic distortion, power quality.

\section{Introduction}

The number of power electronic-based loads such as plugin electric vehicles, photovoltaic inverters, and lamps with electronic ballast in modern distribution networks is continuously increasing. These electrical appliances are loads with a nonlinear voltage-current characteristic. Therefore, even if the supply voltage at the connection point represents a purely sinusoidal waveform, the current flowing through the load is distorted and thus, its waveform is not purely sinusoidal. Furthermore, the distorted current causes in turn a distortion of the voltage due to a voltage drop over the harmonic network impedance [1].

With the widespread use of nonlinear loads in distribution networks, it is becoming more important to model the characteristics of harmonic generation in order to perform harmonic studies in real low voltage (LV) networks. Considering the computational performance to determine the harmonic contents of a nonlinear load, a harmonic analysis based on frequency domain models is preferable to a harmonic analysis based on time-domain models [2]. In literature different models for a harmonic analysis of a nonlinear load in frequency-domain are described [3], [4]. Among these models, a constant current source model, an uncoupled Norton equivalent model and a coupled Norton equivalent model are introduced as the commonly preferred models [5]. The constant current source model is often chosen for large-scale network simulations due to its simplicity. However, due to the dependency of harmonic currents on the harmonic voltage, the other two models based on a Norton equivalent are expected to give more accurate results. The reason is that these models additionally comprise a harmonic admittance, which accounts for the voltage dependency of the harmonic emission [6], [7]. All of these frequency domain models consist of a set of parameters, which can be derived from either measurements or time-domain simulations of the respective nonlinear load. The accuracy of these models depends both on the nonlinearity of the load and the limits of the required linearization [8]. Usually, a sinusoidal voltage is used as reference point, but it is also possible to use other voltage waveforms for the linearization. Therefore, it is necessary to evaluate the impact of different reference points on the model accuracy [2].

In this paper, a Compact Fluorescent Lamp (CFL) is selected as an example of a strongly nonlinear load and modeled by using three different frequency domain models. The harmonic characterization of the CFL is carried out by using time-domain simulations under different voltage conditions in MATLAB $₫ /$ Simulink $₫$. Then, the parameters of the frequency domain models of the nonlinear load are identified. The harmonic output results of the models are examined for the same voltage conditions. Finally, a comparison of these models is made by determining the relative errors, which illustrate the limitations of the models and their linearization. A main advantage of the simulation-based approach compared to the measurement-based one is that the results are not affected by uncertainties of the measurement equipment. 


\section{Frequency Domain Models}

In this section, a detailed description of the three mentioned frequency domain models is given.

\section{A. Constant Current Source Model}

The parameters of a constant current source model are obtained assuming that there is no change in the operating conditions of the supply system. It is necessary to define a reference voltage, i.e. a voltage source having a certain harmonic spectrum. This voltage source is then directly connected to the terminal of the nonlinear load and subsequently, a measurement or a simulation is performed. As a result, a harmonic spectrum of the resulting current waveform at the load terminal is obtained. This harmonic spectrum represents the parameters of the model. The constant current source model is a simple and easy applicable approach, but it may not provide sufficiently accurate results for a harmonic producing load under considerably changing voltage conditions [6].

\section{B. Uncoupled Norton Equivalent Model}

The uncoupled Norton equivalent model extends the constant current source model and considers the effects of changing voltage conditions on the harmonic currents by using admittances. Two parameters are required for each frequency of interest: a reference harmonic current and an impedance or admittance, respectively [6], [9], [13]. The reference harmonic current is determined in the same way as for the constant current source model. The harmonic admittance is calculated by performing a linearization around the reference voltage [10] [11]. In this paper, the linearization is based on Taylor's theorem using the constant term and the first derivative (with the linear expression $\left.\mathrm{y}=\mathrm{a} \cdot \Delta \mathrm{x}+\boldsymbol{y}_{\mathbf{0}}: \Delta \mathrm{y}=\boldsymbol{y}-\boldsymbol{y}_{\mathbf{0}}, \Delta \mathrm{x}=\boldsymbol{x}-\boldsymbol{x}_{\mathbf{0}}\right)$. The constant value represented by $\boldsymbol{y}_{\mathbf{0}}$ defines the reference point of the linearization. The linearization of a nonlinear function is illustrated in Fig. 1.

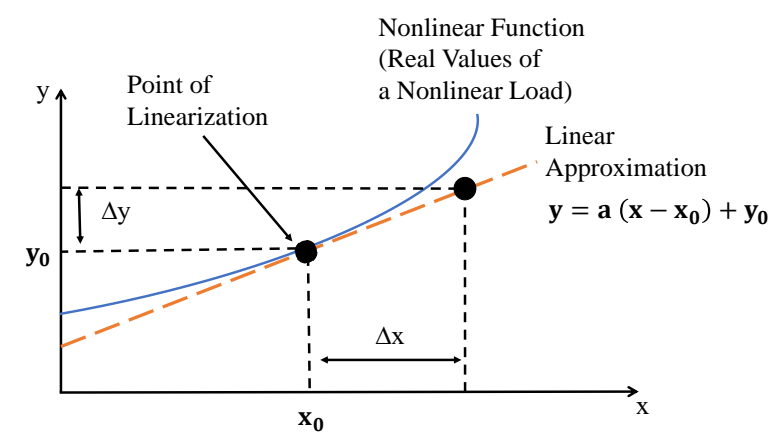

Fig. 1. Linearization of a nonlinear function [2] [17]

The equations of the model are given in the following with (1) being the compact form of (2). All variables are complex phasors.

$$
\underline{\boldsymbol{i}}=\underline{\boldsymbol{Y}} \cdot \underline{\Delta \boldsymbol{v}}+\underline{\boldsymbol{i}}_{\mathrm{ref}}
$$

$$
\left[\begin{array}{c}
\underline{I}_{1} \\
\underline{I}_{2} \\
\underline{I}_{3} \\
\cdot \\
\underline{I}_{\mathrm{M}}
\end{array}\right]=\left[\begin{array}{ccccc}
\underline{Y}_{1,1} & 0 & 0 & \cdot 0 \\
0 & \underline{Y}_{2,2} & 0 & . & 0 \\
0 & 0 & \underline{Y}_{3,3} & \cdot & 0 \\
0 & 0 & 0 & \cdot & \underline{Y}_{\mathrm{M}, \mathrm{M}}
\end{array}\right]\left[\begin{array}{c}
\Delta \underline{V}_{1} \\
\Delta \underline{V}_{2} \\
\Delta \underline{V}_{3} \\
\dot{V_{\mathrm{M}}}
\end{array}\right]+\left[\begin{array}{c}
\underline{I}_{1}^{\mathrm{ref}} \\
\underline{I}_{2}^{\mathrm{ref}} \\
\underline{I}_{-3}^{\mathrm{ref}} \\
\cdot \\
\underline{I}_{\mathrm{M}}^{\mathrm{ref}}
\end{array}\right]
$$

$\underline{\boldsymbol{Y}}$ is the frequency coupling or harmonic admittance matrix and $\underline{\boldsymbol{i}}_{\text {ref }}$ is the harmonic current vector at the reference point. As seen in (2), the model only considers the relation between harmonic voltages and currents of the same order, which corresponds to the diagonal of the shown harmonic admittance matrix [2], [17]. The complex admittance $\underline{Y}_{\mathrm{h}, \mathrm{h}}$ for harmonic order $h(h=1 \ldots N)$ is calculated by (3).

$$
\underline{Y}_{\mathrm{h}, \mathrm{h}}=\frac{\Delta \underline{I}_{\mathrm{h}}}{\Delta \underline{V}_{\mathrm{h}}}=\frac{\underline{I}_{\mathrm{h}}-\underline{I}_{\mathrm{h}}^{\mathrm{ref}}}{\underline{V}_{\mathrm{h}}-\underline{V}_{\mathrm{h}}^{\mathrm{ref}}}
$$

\section{Coupled Norton Equivalent Model}

In addition to the uncoupled Norton equivalent model, this model also considers the couplings between different harmonic orders, e.g. the effects of the fifth harmonic voltage on the third harmonic current. The equation of the model is given in (4). A particular element of $\underline{\boldsymbol{Y}}$ is calculated according to (5).

$$
\begin{gathered}
{\left[\begin{array}{c}
\underline{I}_{1} \\
\underline{I}_{2} \\
\underline{I}_{3} \\
\underline{I}_{\mathrm{M}}
\end{array}\right]=\left[\begin{array}{cccc}
\underline{Y}_{1,1} & \underline{Y}_{1,2} & \underline{Y}_{1,3} & \cdot_{1, \mathrm{~N}} \\
\underline{Y}_{2,1} & \underline{Y}_{2,2} & \underline{Y}_{2,3} & \cdot_{2, \mathrm{~N}} \\
\underline{Y}_{3,1} & \underline{Y}_{3,2} & \underline{Y}_{3,3} & \cdot_{3, \mathrm{~N}} \\
\underline{Y}_{\mathrm{M}, 1} & \underline{Y}_{\mathrm{M}, 2} & \underline{Y}_{\mathrm{M}, 3} & \cdot \underline{Y}_{\mathrm{M}, \mathrm{N}}
\end{array}\right]\left[\left[\begin{array}{c}
\Delta \underline{V}_{1} \\
\Delta \underline{V}_{2} \\
\Delta \underline{V}_{3} \\
\dot{V}_{\mathrm{N}}
\end{array}\right]\right]+\left[\begin{array}{c}
\underline{I}_{1}^{\mathrm{ref}} \\
\underline{I}_{-}^{\mathrm{ref}} \\
\underline{I}_{-3}^{\mathrm{ref}} \\
\cdot \\
\underline{I}_{\mathrm{M}}^{\mathrm{ref}}
\end{array}\right]} \\
\underline{Y}_{\mathrm{k}, \mathrm{h}}=\frac{\underline{I}_{\mathrm{h}}}{\Delta \underline{V}_{\mathrm{k}}}=\frac{\underline{I}_{\mathrm{h}}-\underline{I}_{\mathrm{h}}^{\mathrm{ref}}}{\underline{V}_{\mathrm{k}}-\underline{V}_{\mathrm{k}}^{\mathrm{ref}}}
\end{gathered}
$$

As seen in (4), also the non-diagonal elements, i.e the admittances $\underline{\boldsymbol{Y}}_{\mathbf{k}, \mathbf{h}}$ with $\boldsymbol{k} \neq \boldsymbol{h}$, are required for the parametrization of the coupled Norton equivalent model [6], [12].

\section{Simulation Framework}

In this section, the necessary parameters and simulations of a time-domain model of a CFL are explained.

\section{A. Simulink Model of a CFL}

A compact fluorescent lamp (CFL) without power factor correction (PFC), which represents the commonly used circuit topology for CFLs as well as for many other low power devices, is selected as a nonlinear load. In order to develop the introduced frequency domain models, different voltage sources, namely an AC reference voltage source and an AC distorted voltage source, need to be defined in the Simulink model of the CFL load. While the former serves as a reference, the other is used for generating voltage deviations from that reference. The simulation model of the CFL is shown in Fig. 2 and the parameters of the CFL load are given in Table. 1. 


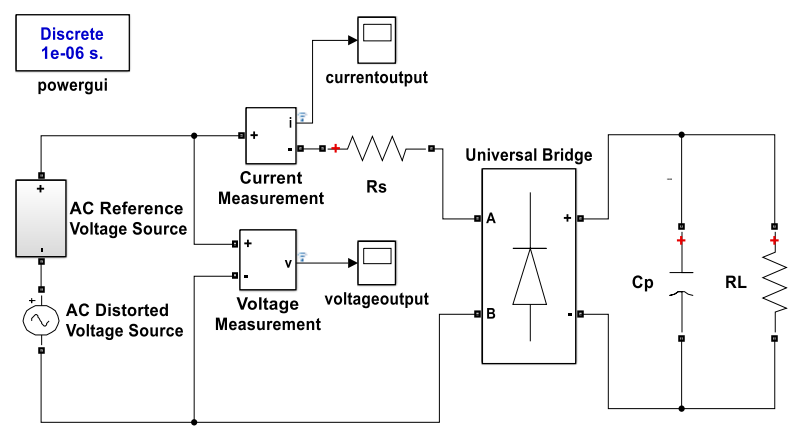

Fig. 2. Simulation model of the CFL (without PFC)

Table I. - Parameters of the selected CFL Load Model [15]

\begin{tabular}{|l|c|}
\hline Rated power & $24 \mathrm{~W}$ \\
\hline $\boldsymbol{R}_{\boldsymbol{s}}:$ Resistor of the rectifying stage & $10 \Omega$ \\
\hline $\boldsymbol{C}_{\boldsymbol{p}}:$ Smoothing capacitor & $5.5 \mu \mathrm{F}$ \\
\hline $\boldsymbol{R}_{\boldsymbol{L}}:$ Resistor of the inverter stage & $3.98 \mathrm{k} \Omega$ \\
\hline
\end{tabular}

\section{B. Simulation Procedure}

The simulation starts using a reference voltage source (with or without harmonic distortion) to determine the reference current required for the frequency domain models. In order to obtain the harmonic admittance matrix (linearization around the reference point) of the uncoupled and coupled Norton equivalent model, the following measurement procedure is carried out for each frequency of interest:

- Superimpose a harmonic voltage on the reference voltage

- Obtain the resulting current and voltage waveforms at the load terminal and select one cycle of each waveform (same time interval)

- Compute harmonic spectrums of the waveforms

- Determine the harmonic admittances of the Norton models and calculate their mean values

The test of individual harmonic is performed according to the fingerprint method described in [16]. All simulations are carried out in discrete-time and the harmonic spectra of the resulting waveforms are computed using the FFT (Fast Fourier Transform) in MATLAB.

\section{Parameter Identification of the Models}

Two reference points are examined: a purely sinusoidal voltage waveform without harmonic distortion and a typical flat-top voltage waveform as given in Table II. Basis for the parametrization is the harmonic current spectrum of the CFL Simulink model at the reference voltage under steadystate conditions. The results are plotted in Fig. 3 and Fig 4.

Table II. - Harmonic spectrum of the selected flat-top voltage waveform (relative to the fundamental voltage)

\begin{tabular}{|c|c|c|}
\hline $50 \mathrm{~Hz}: 325.3 V_{\text {peak }}(230 \mathrm{rms})$ & $100.00 \%$ & $0.0^{\circ}$ \\
\hline $150 \mathrm{~Hz}(\mathrm{~h} 3)$ & $2.37 \%$ & $0.0^{\circ}$ \\
\hline $250 \mathrm{~Hz}(\mathrm{~h} 5)$ & $1.66 \%$ & $180.0^{\circ}$ \\
\hline $350 \mathrm{~Hz}(\mathrm{~h} 7)$ & $0.88 \%$ & $0.0^{\circ}$ \\
\hline $450 \mathrm{~Hz}(\mathrm{~h} 9)$ & $0.24 \%$ & $180.0^{\circ}$ \\
\hline $550 \mathrm{~Hz}(\mathrm{hll})$ & $0.13 \%$ & $180.0^{\circ}$ \\
\hline $650 \mathrm{~Hz}(\mathrm{hl})$ & $0.24 \%$ & $0.0^{\circ}$ \\
\hline $750 \mathrm{~Hz}(\mathrm{hl})$ & $0.16 \%$ & $180.0^{\circ}$ \\
\hline
\end{tabular}

(a)

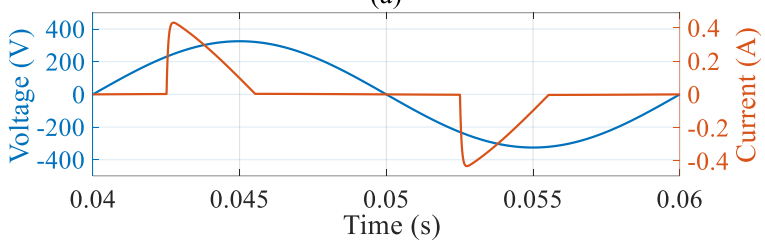

(b)

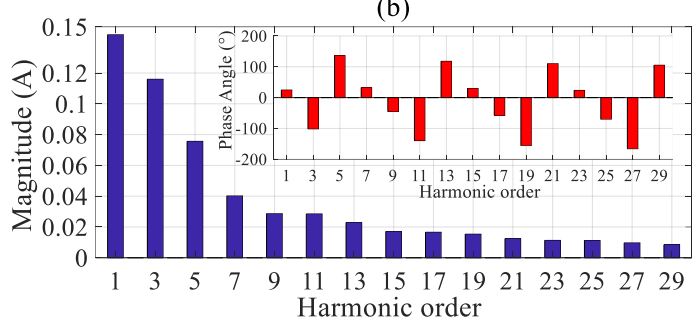

Fig. 3. Sinusoidal voltage: (a) current and voltage waveforms, (b) harmonic current spectrum (peak values)

(a)

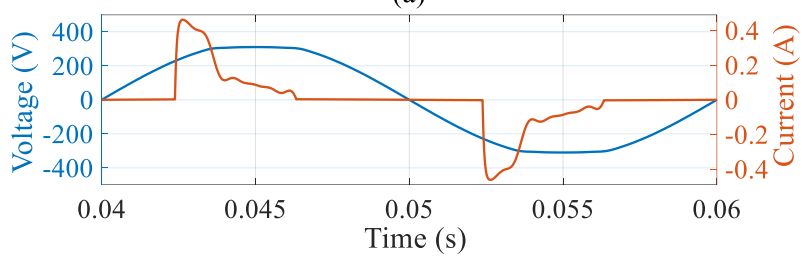

(b)

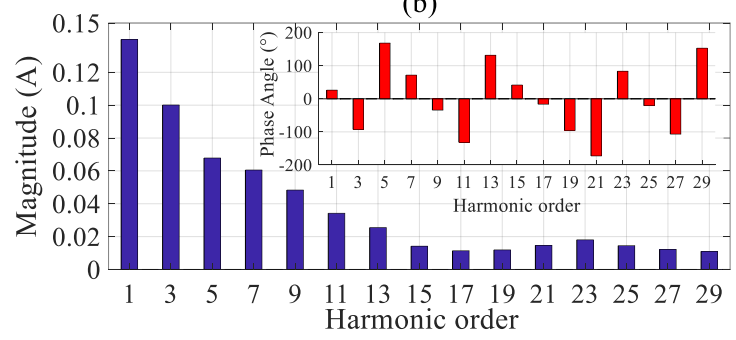

Fig. 4. Flat-top voltage: (a) current and voltage waveforms, (b) harmonic current spectrum (peak values)

The fingerprint graphs of the $3^{\text {rd }}$ harmonic voltage and the corresponding $3^{\text {rd }}$ harmonic current are shown exemplarily in Fig. 5 for both reference voltages. (a)

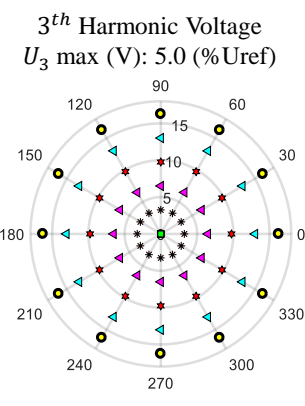

$3^{\text {th }}$ Harmonic Current

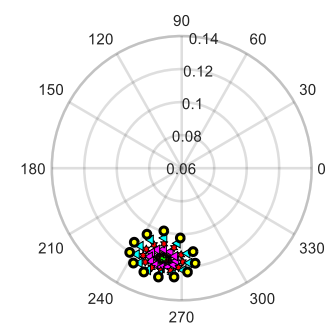
$I_{3,3}$ max Magnitude (A): 0.12 (b)

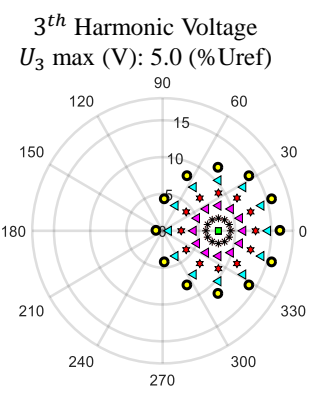

$3^{\text {th }}$ Harmonic Current $I_{3,3} \max$ Magnitude (A): 0.11

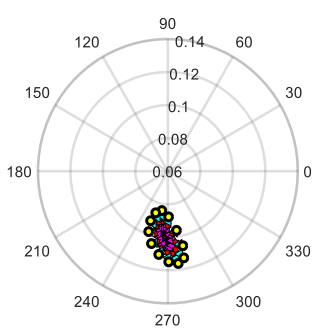

Fig. 5. Fingerprint graphs of the individual $3^{\text {rd }}$ harmonic voltages and currents (a) sinusoidal voltage, (b) flat-top voltage 
The angle of the harmonic voltages is changed in steps of $30^{\circ}$, while the magnitude is increased in five steps until the limit value of the respective harmonic voltage according to EN 50160 is reached. The admittances corresponding to the $3^{\text {rd }}$ harmonic current and a phase angle of $30^{\circ}$ of the tested harmonic voltages are plotted for both reference points in Fig. 6. While the blue arrows indicate the admittances for the sinusoidal reference voltage, the magenta arrows represent the admittances for the flat-top reference voltage. The spread of the admittances in the complex plane is an indicator for the dependence of the admittances on the magnitude of the applied harmonic voltage. There is often also a strong dependence on the phase angle, which is, however, not shown here. The complex mean values of the admittances are used to parameterize the Norton models.
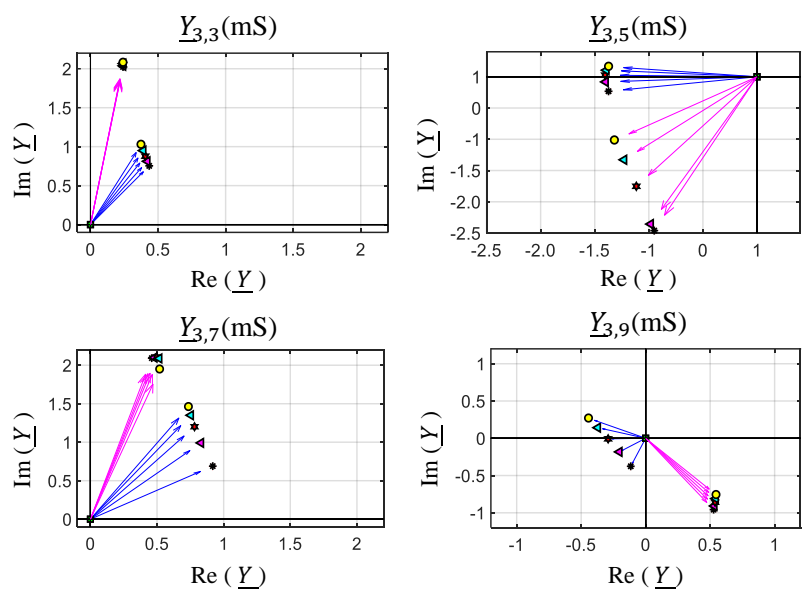

Fig. 6. Admittances corresponding to the $3^{\text {rd }}$ harmonic current and a phase angle of $30^{\circ}$ of the tested harmonics for both reference voltages (blue arrows: sinusoidal, magenta arrows: flat-top)

For a comparative view of the admittances for odd harmonics (up to $25^{\text {th }}$ order), a heat map showing the mean absolute values of the admittances along with their phase angles is given in Fig. 7 for the sinusoidal reference voltage and in Fig. 8 for the flat-top reference voltage, respectively. Both admittance matrices are qualitatively similar, but there are some slight quantitative differences, which occur mainly for the $9^{\text {th }}$ harmonic voltage. This is shown in Fig. 9, which displays the absolute difference of the complex admittances according to (6). $\mathbf{k}$

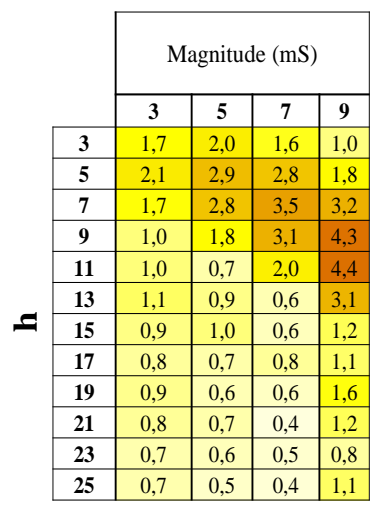

k

\begin{tabular}{|c|c|c|c|c|}
\hline \multicolumn{4}{|c|}{ Phase Angle $\left({ }^{\circ}\right)$} & Color \\
\hline 3 & 5 & 7 & 9 & \\
\hline 52 & -148 & 18 & -150 & 6,4 \\
\hline-79 & 72 & -134 & 28 & 5,2 \\
\hline 159 & -64 & 77 & -137 & 4,4 \\
\hline 62 & 167 & -68 & 71 & 3,3 \\
\hline-10 & 78 & 151 & -74 & 2,9 \\
\hline-111 & 16 & 33 & 147 & 2,5 \\
\hline 148 & -94 & 1 & 32 & 2,1 \\
\hline 65 & 167 & -115 & -7 & 1,7 \\
\hline-26 & 88 & 132 & -120 & 1,3 \\
\hline-126 & -7 & 57 & 124 & 0,9 \\
\hline 144 & -105 & -35 & 53 & 0,5 \\
\hline 57 & 167 & -139 & -34 & 0,2 \\
\hline
\end{tabular}

Fig. 7. Mean absolute values of the admittances $\underline{Y}_{k, h}$ for the sinusoidal reference voltage

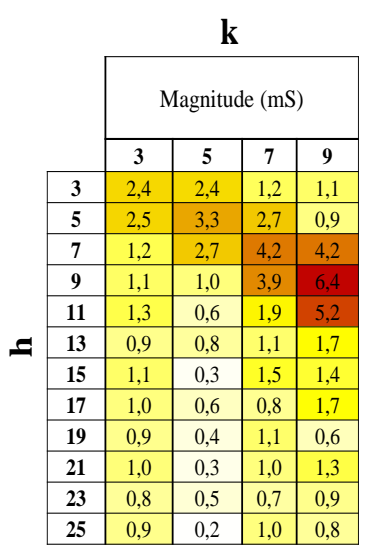

\begin{tabular}{|c|c|c|c|c|}
\hline \multicolumn{4}{|c|}{ Phase Angle $\left({ }^{\circ}\right)$} & \multirow{2}{*}{$\begin{array}{l}\text { Color } \\
\text { Scale }\end{array}$} \\
\hline 3 & 5 & 7 & 9 & \\
\hline 62 & -125 & 63 & -24 & 6.4 \\
\hline-75 & 80 & -125 & 44 & 5,2 \\
\hline 171 & -72 & 71 & -130 & 4,4 \\
\hline 116 & 146 & -82 & 75 & 3,3 \\
\hline 11 & 132 & 143 & -75 & 2,9 \\
\hline-73 & 3 & 92 & 148 & 2,5 \\
\hline-154 & -79 & -16 & 136 & 2,1 \\
\hline 107 & -150 & -112 & 6 & 1,7 \\
\hline 32 & 89 & 177 & -67 & 1,3 \\
\hline-62 & 45 & 68 & -144 & 0,9 \\
\hline-151 & -71 & -3 & 99 & 0,5 \\
\hline 128 & -157 & -99 & 53 & 0,2 \\
\hline
\end{tabular}

Fig. 8. Mean absolute values of the admittances $\underline{Y}_{k, h}$ for the flat-top reference voltage

However, the differences regarding the non-linearity, i.e. the deviations of the admittances from their mean values, can be significantly higher for different reference points. This would result in different model accuracies even for the same magnitude of voltage change $\left|\Delta \underline{V}_{k}\right|$ with respect to the reference.

$$
\Delta \underline{Y}=\left|\underline{Y}_{\text {Sinusoidal }}-\underline{Y}_{\text {Flat-top }}\right|
$$

$\mathbf{k}$

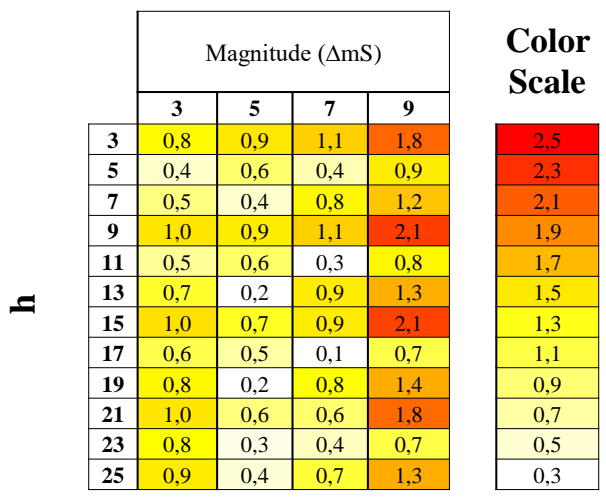

Fig. 9. Absolute difference of the complex admittances between corresponding values of the admittance matrices for both reference voltages

\section{Model Validation}

In order to evaluate the impact of the reference voltage on the model accuracy, the developed frequency domain models, which are based on sinusoidal and flat-top voltage, are compared with the time-domain model in Simulink. The validation process is divided into two parts. While the first part deals with the test of individual harmonic voltages, the second part analyzes the accuracy for typical voltage distortions comprising of various harmonic voltages. The model accuracy of the frequency domain models is characterized by the phase angle deviation $\left(\Delta^{\circ}\right)$, the relative magnitude error (RME) and the total relative error (TRE). With respect to a certain harmonic order $k$, the phase angle deviation is calculated by (7) and the relative magnitude error (RME) is calculated by (8). 


$$
\begin{gathered}
\text { Phase Angle }\left(\Delta^{\circ}\right)=\theta_{\mathrm{k}}^{\text {est }}-\theta_{\mathrm{k}}^{\text {real }}\left(\underline{I}_{\mathrm{k}}=\left|\underline{I}_{\mathrm{k}}\right| \angle \theta_{\mathrm{k}}\right) \\
\operatorname{RME}(\%)=\frac{|| \underline{I}_{\mathrm{k}}^{\text {est }}|-| \underline{I}_{\mathrm{k}}^{\text {real }}||}{\left|\underline{I}_{\mathrm{k}}^{\text {real }}\right|} \cdot 100 \% \\
\operatorname{TRE}(\%)=\frac{\sqrt{\sum_{\mathrm{k}=3}^{9}\left(\left|\underline{I}_{\mathrm{k}}^{\text {est }}-\underline{I}_{\mathrm{k}}^{\text {real }}\right|\right)^{2}}}{\left|\underline{I}_{1}^{\text {real }}\right|} \cdot 100 \%(\mathrm{k}=3,5,7,9)
\end{gathered}
$$

$\underline{I}_{k}^{e s t}$ is the estimated value calculated from the results of a frequency domain model and $\underline{I}_{k}^{\text {real }}$ is the real value taken from the result of the Simulink model of the CFL. The total relative error (TRE) in (9) aggregates the error of several harmonic orders and is defined in a similar way as the THD. However, it is limited to the harmonic currents of the $3^{\text {rd }}$ to the $9^{\text {th }}$ order as only these orders are covered by the models.

\section{A. Individual Harmonics}

The model validation is performed for individual harmonics first. The test points are defined in a similar way as for the fingerprint analysis: The magnitude of each harmonic voltage $\left(3^{\text {rd }}\right.$ to $9^{\text {th }}$ order $)$ is increased in 10 steps until its limit value (acc. to EN 50160) and the phase angle is varied in steps of $60^{\circ}$. The resulting 60 test points for each harmonic are superimposed on the flat-top reference voltage and the deviation to the time-domain simulation is calculated according to (7), (8) and (9) for each test point. The relative magnitude error (RME) and the phase angle deviation $\left(\Delta^{\circ}\right)$ are calculated exemplarily for the $3^{\text {rd }}$ harmonic current. The results for the frequency domain models based on sinusoidal and flat-top voltage are shown in Fig. 10 and Fig. 11, respectively. The "X" axes indicate the tested cases $\left(\boldsymbol{I}_{\mathrm{k}, \mathrm{h}}\right.$, $\mathrm{h}$ : order of the superimposed harmonic voltage, k: order of the calculated harmonic current). Each Box-Whisker plot shows the variation of the results within the 60 test points. The inner limits of the colored rectangle correspond to the $25^{\text {th }}$ and $75^{\text {th }}$ percentile, while the red line in the center represents the median. Besides, the outer black lines indicate the $5^{\text {th }}$ and $95^{\text {th }}$ percentile.
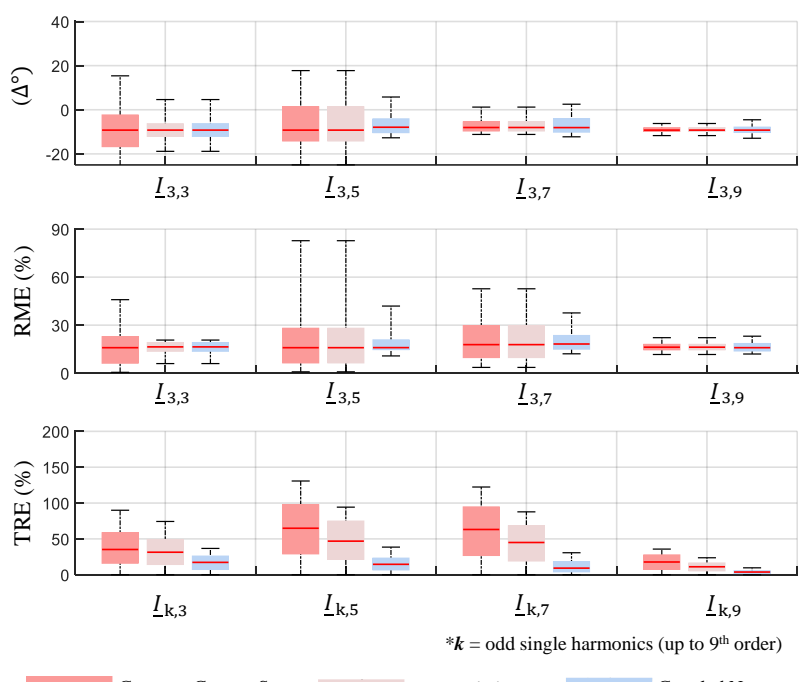

Fig. 10. Model validation for the models based on the purely sinusoidal reference voltage using individual harmonics
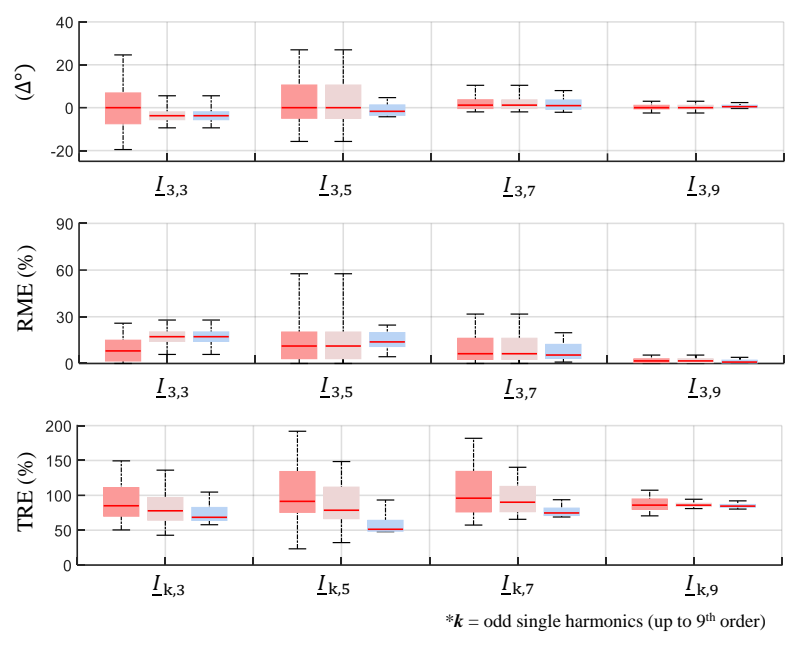

Constant Current Source Uncoupled Norton Coupled Norton

Fig. 11. Model validation for the models based on the flat-top reference voltage using individual harmonics

The coupled Norton model usually provides the best results. As expected, the uncoupled Norton model behaves identical to the coupled Norton model for the relation between harmonics of the same order, while it provides the same results as the constant current source model for harmonics of different order. Regarding the total relative error (TRE), the uncoupled Norton model shows a better accuracy than the constant current source model. However, the most important point is that the overall performance of the models based on flat-top reference voltage is better than that of those using sinusoidal reference voltage. As the models are intended to predict the harmonic levels in real networks, the used test points, which are derived from a typical flat-top voltage, represent a reasonable choice for the model comparison.

\section{B. Typical Voltage Distortions}

The model validation is additionally performed using a set of typical voltage distortions. For that purpose, a set of totally 36 flat-top waveforms is synthetically generated (Fig. 12a). The corresponding harmonic spectra are obtained after applying the Fourier transform. Due to the use of only odd harmonics up to the $9^{\text {th }}$ order, the resulting waveforms differ from the original ones (Fig. 12b).
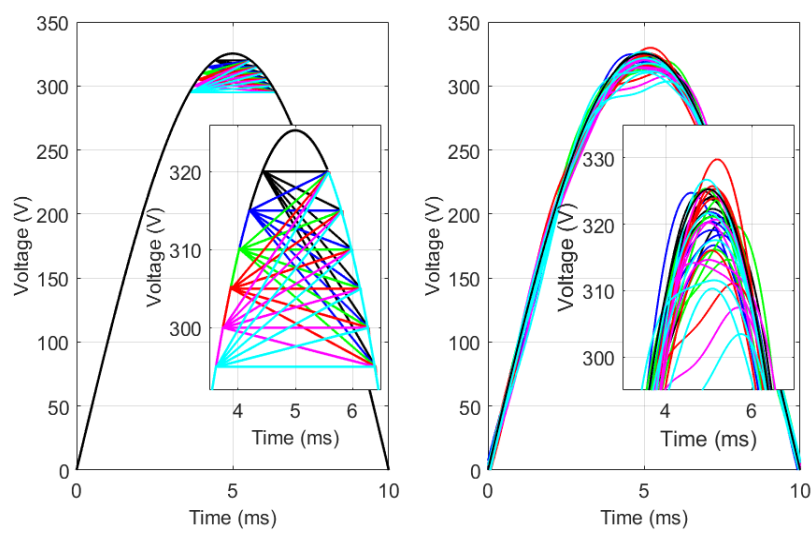

Fig. 12. (a) Created (b) Resulting flat-top voltage waveforms. 
The relative magnitude error (RME) and the phase angle deviation $\left(\Delta^{\circ}\right)$ are exemplarily calculated for the $3^{\text {rd }}$ harmonic current. The total relative error (TRE) is calculated for the considered range of harmonic currents (odd harmonics up to the $9^{\text {th }}$ order). Fig. 13 shows the results of the accuracy analysis for the frequency domain models using the same Box-Whisker representation as in Fig. 10.
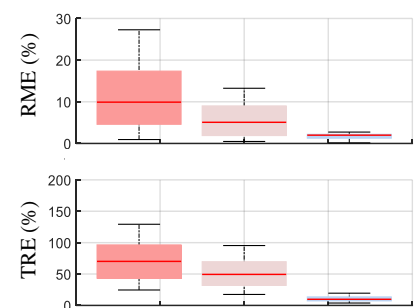

Constant Current Soutce
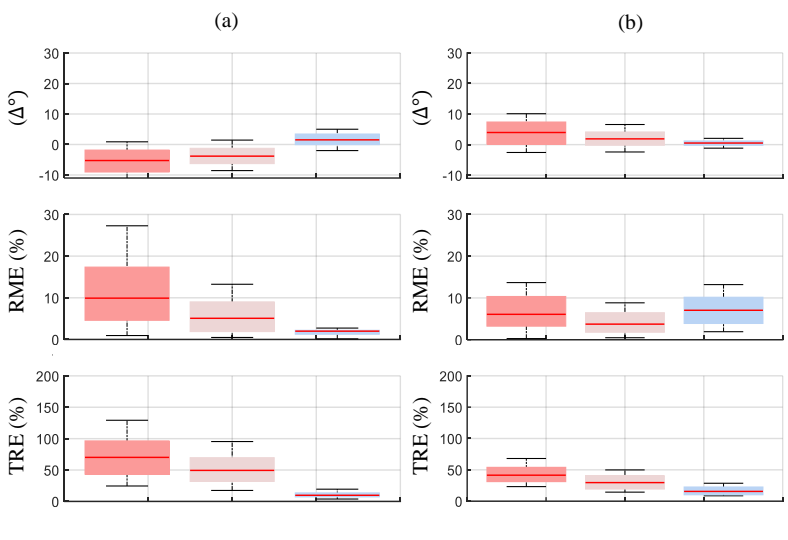

Fig. 13. Model validation of the frequency domain models using typical voltage distortions ( $\operatorname{RME}(\%)$ and $\left(\Delta^{\circ}\right)$ with $3^{\text {rd }}$ harmonic);

(a) sinusoidal reference voltage, (b) flat-top reference voltage.

The coupled Norton model usually provides a better estimation of the harmonic current emission than the other models. Its accuracy is only slightly influenced by the used reference voltage. However, the accuracy of the constant current source model as well as the accuracy of the uncoupled Norton model can be significantly improved if they are parameterized using the flat-top reference voltage.

\section{Conclusion}

The paper compares the results of three commonly used frequency domain models for the estimation of the harmonic current emission of a CFL. The parameters of the frequency domain models are identified from the results of a time-domain simulation of a CFL Simulink ${ }^{\circledR}$ model by performing simulations under two reference voltages: a purely sinusoidal and a typical flat-top voltage waveform.

The results of the model validation using individual harmonics show that the model accuracy depends strongly on the reference point used for the linearization. In typical LV networks it can be expected that the models based on flat-top reference voltage provide better results. This is also shown when typical voltage distortions are used for the validation. Particularly, the constant current source model and the uncoupled Norton model provide much better results if they are parameterized using the flat-top voltage.

Further similar studies should be carried out for various nonlinear loads including different topologies, e.g. power supplies with a passive power factor correction. In addition, the parameters of the frequency domain models (especially for the constant current source and the uncoupled Norton model) can be determined under various flat-top reference voltage conditions for improving their accuracies.

\section{References}

[1] Bollen, M.H. and Gu, I.Y., Signal Processing Of Power Quality Disturbances, volume 30. John Wiley \& Sons, 2006, pp. 122-130.

[2] J. E. Caicedo, A. A. Romero, R. Langella, J. Meyer, N. R. Watson, "Impact of Reference Conditions on the Frequency Coupling Matrixof a Plug-in Electric Vehicle Charger", published to 18th ICHQP, Ljubljana, Slovenia, May 2018.

[3] A. Medina, J. Segundo-Ramirez, P. Ribeiro, W. Xu, K. L. Lian, G. W. Chang, V. Dinavahi, and N. R. Watson, "Harmonic Analysis in Frequency and Time Domain", IEEE Transactions on Power Delivery, Vol. 28, No. 3, July 2013.

[4] J. Meyer, S. Müller, P. Schegner, S. Z. Djokic, A. J. Collin, and $\mathrm{X} . \mathrm{Xu}$, "Comparison of Methods for Modelling Electric Vehicle Chargers for Harmonic Studies", 2016 Power Systems Computation Conference (PSCC), June 2016.

[5] J.E. Caicedo, A. A. Romero, H. C. Zini, "Assessment of the Harmonic Distortion in Residential Distribution Networks: Literature Review", Ingeniería e Investigación vol. 37 n: 3, December - 2017 (72-84).

[6] C.F.M. Almeida, N. Kagan, "Harmonic Coupled Norton Equivalent Model for Modeling Harmonic-Producing Loads", 14th International Conference on Harmonics and Quality of Power - ICHQP 2010.

[7] A.B. Nassif, J. Yong, W. Xu, "Measurement-based Approach for Constructing Harmonic Models of Electronic Home Appliances", IET Generation, Transmission \& Distribution Received on 15th December 2008.

[8] E. O. de Matos, T. M. Soares, U. H. Bezerra, M. E. L. Tostes, A. R. A. Manito, and B. C. Costa Jr, "Using Linear And Nonparametric Regression Models to Describe the Contribution of Nonlinear Loads on the Voltage Harmonic Distortions in the Electrical Grid," IETGeneration transmition and distribuition, Vol. 10, Issue: 8, 5192016.

[9] E. Thunberg, L. Söder, "A Norton Approach to Distribution Network Modeling for Harmonic Studies", IEEE Transactions on Power Delivery. Volume: 14, Issue: 1 , Jan 1999.

[10] K. M. Hangos, J. Bokor, G. Szederkényi, Analysis and Control of Nonlinear Process Systems. Springer-Verlag London, pp. 1-308 (2004).

[11] E.V. Larsen, D.H. Baker, J.C. McIver, "Low-order harmonic interactions on $\mathrm{AC} / \mathrm{DC}$ systems", IEEE Transactions on Power Delivery. Vol. 4, Issue: 1, Jan 1989.

[12] E. Thunberg ; L. Soder, "Influence of the Network Impedance on Distribution System Harmonic Norton Models", PowerCon 2000. 2000 International Conference on Power System Technology.

[13] J. Arrillaga, B. C. Smith, N. R. Watson, and A. R. Wood, "Power System Harmonic Analysis," New York: Wiley, 1997.

[14] J.E. Caicedo, A. A. Romero, H. C. Zini, "Frequency Domain Modeling of Nonlinear Loads, Considering Harmonic Interaction", IEEE Workshop on Power Electronics and Power Quality Applications (PEPQA), Colombia, July 2017.

[15] A. M. Blanco, S. Yanchenko, J. Meyer, P. Schegner, "Impact of supply voltage distortion on the current harmonic emission of non-linear loads", DYNA, 82 (192). pp. 150-159. ISSN 2346-2183.

[16] J.F.G. Cobben, W.L. Kling, J.M.A. Myrzik, "The making and purpose of a harmonic fingerprint", CIRED 2007.

[17] L. Frater, "Light flicker and harmonic modeling of electrical lighting", Ph.D. dissertation. University of Canterbury, 2015. 